\title{
Handwriting Analysis for Diagnosis and Prognosis of Parkinson's Disease
}

\author{
Atilla Ünlü ${ }^{1}$, Rüdiger Brause ${ }^{1}$, Karsten Krakow ${ }^{2}$ \\ ${ }^{1}$ Institute of Informatics, Johann Wolfgang Goethe-Universität, \\ 60054 Frankfurt a.M., Germany \\ \{Atilla, RBrause\}@informatik.uni-frankfurt.de \\ ${ }^{2}$ Neurological Clinics, Johann Wolfgang Goethe-Universität, \\ Theodor-Stern-Kai 7, 60596 Frankfurt a. M. \\ k.krakow@em.uni-frankfurt.de
}

\begin{abstract}
At present, there are no quantitative, objective methods for diagnosing the Parkinson disease. Existing methods of quantitative analysis by myograms suffer by inaccuracy and patient strain; electronic tablet analysis is limited to the visible drawing, not including the writing forces and hand movements. In our paper we show how handwriting analysis can be obtained by a new electronic pen and new features of the recorded signals. This gives good results for diagnostics.
\end{abstract}

Keywords: Parkinson diagnosis, electronic pen, automatic handwriting analysis

\section{Introduction}

The Parkinson disease is a degenerative disorder of the central nervous system that affects the control of muscles, and so may affect movement, speech and posture [1]. It is often characterized by muscle rigidity, tremor, a slowing of physical movement (bradykinesia), and in extreme cases, a loss of physical movement (akinesia). The primary symptoms are the results of irregular muscle contraction, caused by the insufficient formation and action of dopamine, which is produced in the dopaminergic neurons (substantia nigra) of the brain.

From the pathological view of the Parkinson disease there is no reliable method for an objective, quantitative diagnosis. Clinically, one distinguishs the tremor between type I Parkinson tremor of 4-7 Hz, which can be observed when the hand is not moving, but shaking, and a higher frequency type II Parkinson tremor of $5-12 \mathrm{~Hz}$ which is observed during movement [2].

There are two methods known for quantitative analysis of tremors. Classically, the muscle activity is recorded from electrodes (skin surface electrodes, or steel needles pinned into the muscle through the skin), and printed in the form of myograms EMG [3]. This is not only a tedious procedure for the patient, but also not very accurate and therefore not used in the normal case. The second method has been pioneered by Marquard and Mai [4] and had been widely accepted by research [5],[6]. The 
procedure records the handwriting of the patient on a graphical tablet, especially loops like ellell. These loops are slightly deformed by patients with tremor. The deformation can be used as a feature for diagnostics.

The theory that lies behind this, says that normal handwriting is marked by automation; the movements are so fast that normal feedback loop by visual perception and muscle control is disabled. This results in an open loop configuration. For Parkinson patients, the automation is no longer valid; their handwriting depends on the visual closed loop.

This approach is questioned by researchers who claim that for Parkinson tremor not the absolute positioning but the grip forces are important [7][8][9]. Thus, a force sensitive device is needed for recording the pressure and not the pen location. This is provided by an electronic pen. Since there are no known features for pressure analysis of Parkinson handwritings, in this paper we investigate different kind of features and characterize them by their diagnostic value.

In all experiments, an electronic pen was used for recording the samples for our analysis.

\section{The Electronic Pen Recordings}

Let us first introduce how the data were obtained.

\subsection{The Electronic Pen}

The recording device is an electronic pen, composed of several sensors and integrating a real writing ball pen which was build by the BiSP-Project [10]. This design gives the user the feeling of a real ball pen and facilitates its use by patients. In Fig. 1 the pen architecture and the pen usage are shown.
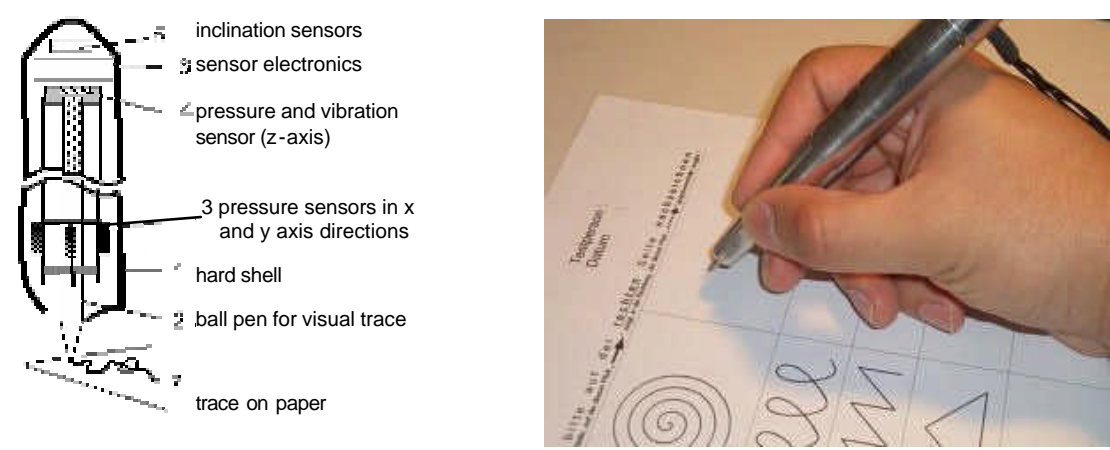

Fig. 1. (a) The architecture of the electronic pen (b) The test writing and recording

In difference to graphic tablets, the recording does not record the absolute position, but only the pressure in $\mathrm{x}, \mathrm{y}$ and $\mathrm{z}$ direction. Additionally, there are two tilt sensors which measure the inclination relative to the $x-y$ plane. 
The writing signals are sampled with a sample frequency of $\mathrm{f}=500 \mathrm{~Hz}$ and cut off at $200 \mathrm{~Hz}$.

\subsection{The Recordings}

In Fig. 2(a), a sample drawing and in Fig. 2(b) its corresponding pressure signals x,y,z and tilt signals $\alpha$ and $\beta$ are shown.

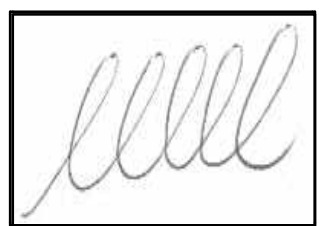

Fig. 2. (a) Handwriting sample

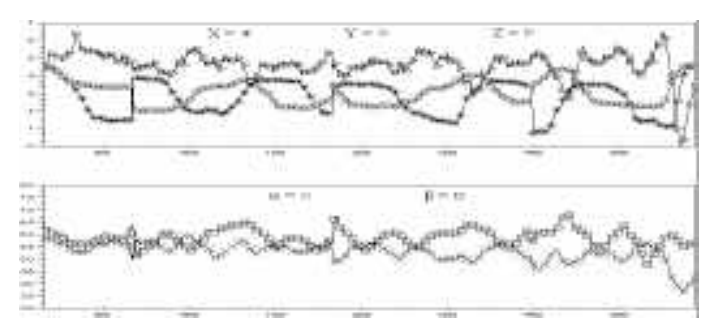

(b) The corresponding recordings of $3 \mathrm{D}$ pressure and tilt

For 28 Parkinson patients and 28 control persons we recorded different patterns: the ellell loops, meanders, words and a complete sentence. As preprocessing, all signal noise and artifacts are filtered by sinc filters.

For the loop analysis, we selected one segment of $\mathrm{N}=1000$ samples from the recordings of each individual. The same segment was tested by different features.

\section{The analysis methods and results}

Our analysis concentrated on finding different relevant features for a high separation of the handwriting of Parkinson patients from those of control persons. Since most of the patients received a medical treatment which compensated the tremor, the signal energy in the high frequency band was not a salient feature for itself. Instead, we had to devise more sophisticated features.

The evaluation of the chosen features was done by a receiver operating characteristic (ROC) analysis in order to characterize the diagnostic possibilities not only by their sensitivity, but also by the specifity of the diagnostic approach. For this, the diagnosis $D(u)$ of feature $u(t)$ was defined by

$$
\mathrm{D}(\mathrm{u})= \begin{cases}\text { ill } & \mathrm{u} \geq \theta \\ \text { not ill } & \mathrm{u}<\theta\end{cases}
$$

The threshold $\theta$ was chosen in discrete steps over the whole range of the feature variable.

The first attempt tried to check the validity of the criteria described in literature. 


\subsection{Relative number of loop extremes}

As criteria we chose the relative number of loop extremes $e$ which were already defined in the literature for tablet handwriting input, see [4]. Here, people with Parkinson tremor tend to have more extremes due to a more irregular movement at writing loops. The number of extremes $e$ depends heavily on the bandwidth of the signal. The increase in $\mathrm{e}\left(\mathrm{f}_{\mathrm{g}}\right)$ with increasing frequency $\mathrm{f}_{\mathrm{g}}$ in signals $x$ and $y$ is more rapid for Parkinson patients than for control people. Therefore, for detection we might use the increase from frequency $f_{1}=1 \mathrm{~Hz}$ to frequency $\mathrm{f}_{30}=30 \mathrm{~Hz}$ reflected by the quotients

$$
\begin{aligned}
\mathrm{x}_{\mathrm{e}}=\left\langle\mathrm{e}_{\mathrm{x}}\left(\mathrm{f}_{30}\right) / \mathrm{e}_{\mathrm{x}}\left(\mathrm{f}_{1}\right)\right\rangle, & \mathrm{y}_{\mathrm{e}}=\left\langle\mathrm{e}_{\mathrm{y}}\left(\mathrm{f}_{30}\right) / \mathrm{e}_{\mathrm{y}}\left(\mathrm{f}_{1}\right)\right\rangle, \\
\alpha_{\mathrm{e}}=\left\langle\mathrm{e}_{\alpha}\left(\mathrm{f}_{30}\right) / \mathrm{e}_{\alpha}\left(\mathrm{f}_{1}\right)\right\rangle, & \beta_{\mathrm{e}}=\left\langle\mathrm{e}_{\beta}\left(\mathrm{f}_{30}\right) / \mathrm{e}_{\beta}\left(\mathrm{f}_{1}\right)\right\rangle .
\end{aligned}
$$

The feature $u_{1}$ is then defined by the averages of pressure signal behaviour to tilt signal behaviour

$$
\mathrm{u}_{1} \equiv \frac{\mathrm{x}_{\mathrm{e}}+\mathrm{y}_{\mathrm{e}}}{\alpha_{\mathrm{e}}+\beta_{\mathrm{e}}}
$$

This gives us an area under the ROC curve of $\mathrm{AUC}=0.896$. The corresponding ROC is shown in Fig. 3

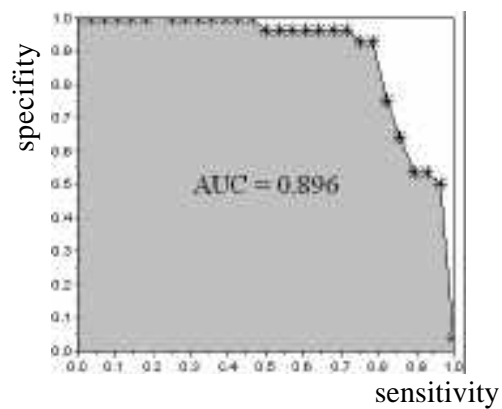

Fig. 3. The receiving operation characteristic for feature $\mathrm{u}_{1}$.

Choosing directly the quotient of extreme values of pressure and tilt for a fixed frequency, say $\mathrm{f}=1 \mathrm{~Hz}$, gives us feature $\mathrm{u}_{2}$

$$
u_{2} \equiv\left\langle\frac{e_{\alpha}(f) \cdot e_{\beta}\left(f_{1}\right)}{e_{x}\left(f_{1}\right)}\right\rangle
$$

This has an AUC $=0.933$ which is even better than that of the first, more complicated feature. 


\subsection{The impulse correlation coefficient}

Regular loops of healthy people tend to be sine-like functions. If we take the derivative of it, the product $-\mathrm{s}(\mathrm{t}) \cdot \mathrm{s} "(\mathrm{t})$ will become maximal. For irregular loops of ill patients, this will decrease. This idea gives us a new feature $\mathrm{s}$ which uses the definition of the impulse correlation coefficient $p_{s 1 s 2}$ of two signals $\mathrm{s}_{1}(\mathrm{t})$ and $\mathrm{s}_{2}(\mathrm{t})$

$$
\mathrm{p}_{\mathrm{s}_{1} \mathrm{~s}_{2}}^{\mathrm{E}}=\frac{\sum_{\mathrm{t}=-\infty}^{\infty} \mathrm{s}_{1}(\mathrm{t}) \cdot \mathrm{s}_{2}(\mathrm{t})}{\sqrt{\mathrm{E}_{\mathrm{s}_{1}} \cdot \mathrm{E}_{\mathrm{s}_{2}}}}
$$

with energies $E_{\mathrm{s} 1}$ and $E_{s 2}$ of the signals. The new feature is then defined for the tilt $\alpha(t)$. Since we are not interested in the offset but in the dynamical behaviour, we take not $\alpha$ and $\alpha^{\prime \prime}$ but their derivatives $\alpha^{\prime}$ and $\alpha^{\prime \prime \prime}$ which produces an impulse correlation coefficient of -1 instead of +1 as best value

$$
\mathrm{u}_{3} \equiv \frac{\sum_{\mathrm{t}=-\infty}^{\infty} \alpha^{\prime}(\mathrm{t}) \cdot \alpha^{\prime \prime \prime}(\mathrm{t})}{\sqrt{\mathrm{E}_{\alpha^{\prime}} \cdot \mathrm{E}_{\alpha^{\prime \prime}}}} .
$$

Using the tilt $\alpha$ gives the best value from the set of all five sensor signals and an AUC $=0.86$. Compared to our first two features this result is not so good. So, let us investigate another set of features by a completely different idea.

\subsection{Approximative entropy}

The tremor signals with their irregularities have some similarities to chaotic signals investigated in chaos theory. There, a measure called "approximative entropy" has been introduced [11]. It takes advantage of the fact that very regular signals of automated movements have a high self-similar degree, but those of irregular movements have not. The number of similar points can be counted and gives a occurrence frequency. The average of the logarithm of this gives us an entropy or average information of the time series. The higher the entropy, the more irregular are the movements.

The computation starts with a sliding window of $m$ samples of signal $s(t)$, giving tuples

$$
\mathbf{r}(\mathrm{i}) \equiv(\mathrm{s}(\mathrm{i}), \mathrm{s}(\mathrm{i}+1), \ldots, \mathrm{s}(\mathrm{i}+\mathrm{m}-1))
$$

The relative number of tuples which are not so different, i.e. which have all entries with a difference smaller than a given number $\varepsilon$, is

$$
C_{i}^{m}(\varepsilon)=\frac{\mid\{(j, k) \mid(|\mathbf{r}(i+k-1)-\mathbf{r}(j+k-1)|) \leq \varepsilon \text { for } k=1 . . m, j=i . . N-m\} \mid}{N-m+1}
$$

This is called a correlation integral. The averaged logarithm is 


$$
\Phi_{\mathrm{m}}(\varepsilon)=\frac{1}{\mathrm{~N}-\mathrm{m}+1} \sum_{\mathrm{i}=1}^{\mathrm{N}-\mathrm{m}+1} \log \mathrm{C}_{\mathrm{i}}^{\mathrm{m}}(\varepsilon)
$$

Then, the approximative entropy of a time series of $N$ samples is defined as the change (tangent) of the entropy due to a variation in the sliding window width $m$

$$
\operatorname{ApEn}(\mathrm{m}, \varepsilon, \mathrm{N})=\Phi_{\mathrm{m}}(\varepsilon)-\Phi_{\mathrm{m}+1}(\varepsilon)
$$

For $\mathrm{m}=2, \varepsilon=3$ and $\mathrm{N}=4$ the ApEn of the primary five signals were computed as demonstration. In Fig. 4 a sample plot of the $x$ signal of a control person writing loops and its $A p E n$ are shown. The $x$ pressure signal has been filtered before with a cut off frequency of $100 \mathrm{~Hz}$.

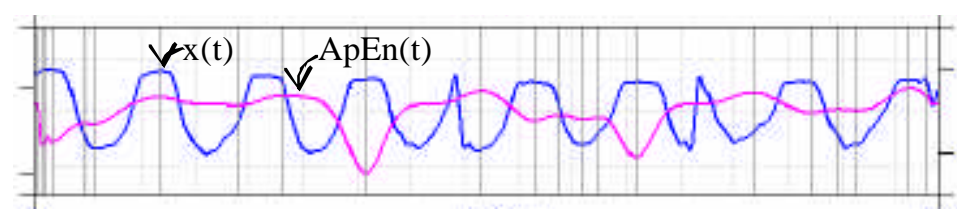

Fig. 4. x-recording of a llelll loop signal and its corresponding ApEn.

For computing the feature value of a handwriting, the whole time series $\mathrm{N}=1000$ and $\varepsilon$ $=12$ were used. As good feature we found

$$
\mathrm{u}_{4} \equiv \frac{\operatorname{ApEn}(\mathrm{z}) \cdot \operatorname{ApEn}(\alpha)}{\operatorname{ApEn}(\mathrm{x}) \cdot \operatorname{ApEn}(\mathrm{y})}
$$

This feature $\mathrm{u}_{4}$ gives us a AUC of 0.905. Although this is not bad, it is still worse than the second feature $\mathrm{u}_{2}$.

\subsection{Multiscale entropy}

A remarkable variant of the approximative entropy is the entropy behaviour due to different time scales. Irregular signals can be characterized by a time scaled entropy analysis called multiscale entropy [12]. This obtained by first computing the average and then the logarithm of the correlation integral of eq.(7)

$$
\Theta(m, \varepsilon, N)=\log \left(\frac{1}{N-m+1} \sum_{i=1}^{N-m+1} C_{i}^{m}(\varepsilon)\right)
$$

The change in this entropy of averaged probabilities due to changing the window width $\varepsilon$ is called sample entropy $\mathrm{S}_{\mathrm{E}}($.

$$
\mathrm{S}_{\mathrm{E}}(\mathrm{m}, \varepsilon, \mathrm{N})=\Theta(\mathrm{m}, \varepsilon, \mathrm{N})-\Theta(\mathrm{m}+1, \varepsilon, \mathrm{N})
$$


We might merge several samples of a time series $s(t)$ together into one sample of a new time series $r(t)$. For instance, for a scale factor of $\tau=3$ we average every three samples into one by

$$
\left.r_{3}(i)=\frac{s(t)+s(t+1)+s(t+2)}{3} \text { with } i=0, \ldots, L N / 3\right\rfloor-1 \quad t=3 i
$$

For every scaled time series with scale $\tau$, the sample entropy $\mathrm{S}_{\mathrm{E} \tau}($.$) can be computed.$ The resulting time series shows if there are irregular, more chaotic components which will remain also in higher time scales. In Fig. 5 the different behaviour of natural signals which have a $1 / \mathrm{f}$ characteristic to not natural ones, e.g. white noise.

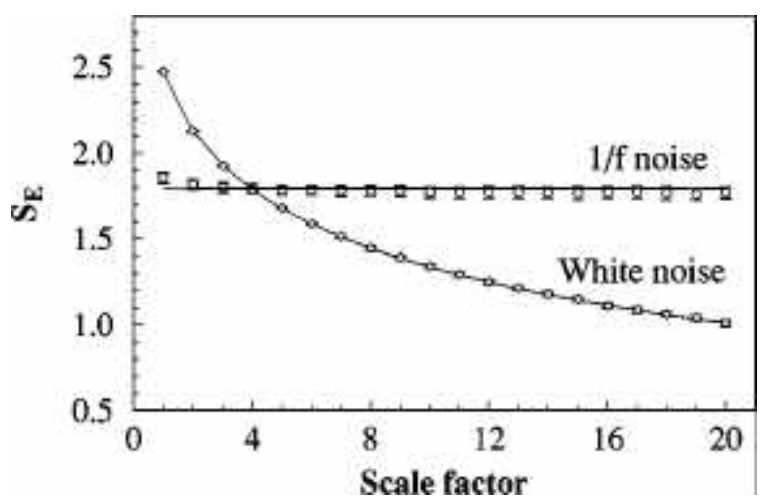

Fig. 5. The multiscale entropy of $1 / \mathrm{f}$ noise and white noise (after [12])

We reflect the different signal slopes by the difference $S_{E}(\tau=4)-S_{E}(\tau=1)$. As new feature $\mathrm{u}_{5}$, let us define the ratio of the pressure behavior to the tilt behavior

$$
\mathrm{u}_{5} \equiv \frac{\mathrm{S}_{\mathrm{E}}(\mathrm{x}, \tau=4)-\mathrm{S}_{\mathrm{E}}(\mathrm{x}, \tau=1)}{\left(\mathrm{S}_{\mathrm{E}}(\alpha, \tau=4)-\mathrm{S}_{\mathrm{E}}(\alpha, \tau=1)\right)\left(\mathrm{S}_{\mathrm{E}}(\beta, \tau=4)-\mathrm{S}_{\mathrm{E}}(\beta, \tau=1)\right)}
$$

All signals were filtered by a $100 \mathrm{~Hz}$ windowed sinc cut off filter before processing, and $S_{E}$ was computed with $m=3$ and $\varepsilon=2$, using the $N$ samples of the scaled time series.

The resulting ROC was not very smooth (see Fig. 6 ) and the corresponding AUC = 0.859 not very convincing. 


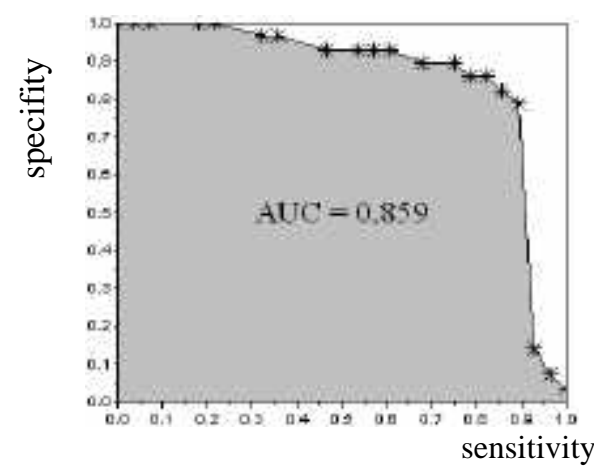

Fig. 6. The ROC of the multiscale feature $\mathrm{u}_{5}$

Therefore, we looked for a more intrinsic discriminating feature for handwriting between Parkinson patients and control persons.

\subsection{Writing power rate coefficient}

One of the most apparent characteristics of the Parkinson disease is the muscle rigidity and tremor. Compared to control persons who have a highly optimized and automated handwriting, Parkinson patients use remarkably more effort and stress for compensating their tremor while producing readable handwriting. This results in less power for the handwriting task.

For the meander pattern we got the mean values of the pressure signals and then computed the signal energy per time unit

$$
\mathrm{u}_{6} \equiv\left(\frac{1}{\mathrm{~N}} \sum_{\mathrm{t}=1}^{\mathrm{N}}(\mathrm{x}(\mathrm{t}))^{2}+\frac{1}{\mathrm{~N}} \sum_{\mathrm{t}=1}^{\mathrm{N}}(\mathrm{y}(\mathrm{t}))^{2}\right)^{-1}
$$

This feature gave an only moderate AUC of 0.84 .

\subsection{Combining the features}

Now, since we have found no ideal feature, what a about combining the most successful ones? Since they have different information sources, do this multiexpert team allow a better diagnosis? The AUC values provides us with a ranking of the possible candidates of $\mathrm{u}_{2}, \mathrm{u}_{4}, \mathrm{u}_{1}, \mathrm{u}_{3}, \mathrm{u}_{5}, \mathrm{u}_{6}$. In fact, taking the best ones gives us a new multi-expert feature

$$
\mathrm{u}_{7} \equiv \mathrm{u}_{2} \mathrm{u}_{4} \mathrm{u}_{1}=\frac{\mathrm{e}_{\alpha}\left(\mathrm{f}_{1}\right) \cdot \mathrm{e}_{\beta}\left(\mathrm{f}_{1}\right)}{\mathrm{e}_{\mathrm{x}}(\mathrm{f})} \cdot \frac{\operatorname{ApEn}(\mathrm{z}) \cdot \operatorname{ApEn}(\alpha)}{\operatorname{ApEn}(\mathrm{x}) \cdot \operatorname{ApEn}(\mathrm{y})} \cdot \mathrm{u}_{1}
$$

Since the quotient $\operatorname{ApEn}(z) / e_{x}\left(f_{1}\right)$ seemed to be roughly constant for our data, we simplified this to 


$$
\mathrm{u}_{8} \equiv \mathrm{e}_{\alpha}\left(\mathrm{f}_{1}\right) \cdot \mathrm{e}_{\beta}\left(\mathrm{f}_{1}\right) \cdot \frac{\operatorname{ApEn}(\alpha)}{\operatorname{ApEn}(\mathrm{x}) \cdot \operatorname{ApEn}(\mathrm{y})} \cdot \mathrm{u}_{1}
$$

obtaining a very nice ROC (see Fig. 7 ) and a very good AUC of 0.963.

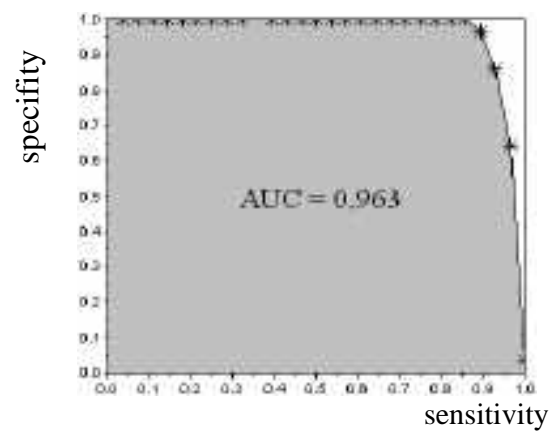

Fig. 7. The ROC of the multi-expert feature $u_{8}$

\section{Discussion}

For the quantitative analysis of muscle anomalies, especially those of Parkinsons`s disease, there are not many analytic possibilities. Here, we discussed a new method based on pressure and movement features recorded by handwriting with an electronic pen. We evaluated different approaches, some of them based on the tremor characteristics for position pressure and tilt of the pen, and some based on methods for quantification of chaotic signals .

It turned out that the most salient feature $\left(\mathrm{u}_{2}\right)$ is based on the difference between the controlled writing pressure in $x-y$ direction and the tilt tremor of the pen. It seems that for these medicated patients the tremor control is better achieved for movements (handwriting) than for constant pressure (pen tilt). The handwriting of Parkinson patients itself have less chaotic characteristics than suspected. Nevertheless, combining all features gives promising indications for diagnosis.

Our results gives a base for quantitative evaluation of the state of a Parkinson patient. This can be used in several fashions.

- The features my be used individually in a long term quantitative recording for the treating doctor in order to detect and predict long term changes in the individual disease history.

- Another application my be the adjustment of the medication regime. The quantitative, easy and quick diagnosis provide a basis for a frequent estimation of the proper drug dosage getting the desired effects and avoiding unwanted side effects. 
- By a very sensitive diagnosis, Parkinson symptoms may be detected very early on persons with a high risk background. This can be used for early medication avoiding subsequent development of the disease.

- Subsequent studies may use these feature not only for a quantitative refinement of a already stated principal diagnosis "Parkinson's disease", but for a diagnosis discriminating general tremor symptoms from special Parkinson ones.

In conclusion, this study showed that there are several features available for good Parkinson diagnosis which can be obtained by simple, cheap and noninvasive handwriting measurements.

Acknowledgments. We want thank all people who have supported us by their work, especially the BiSP team, university of applied sciences Regensburg, Dr. Kessler of the Maria-Hilf clinic, Mönchengladbach, and Dr. Korchounov of the Parkinson clinic, Bad Nauheim.

\section{References}

1. Rajesh Pahwa, Kelly E. Lyons, William C. Koller: Handbook of Parkinson's Disease: Neurological Disease \& Therapy, Marcel Dekker Inc, $3^{\text {rd }}$ ed., New York 2003

2. Andres Ceballos-Baumann, Bastian Conrad: Bewegungsstörungen, Georg Thieme Verlag Stuttgart 2005

3. Roberto Merletti, Philip M. Parker: Electromyography: Physiology, Engineering and Non-Invasive Applications, John Wiley \& Sons Inc, New York 2004

4. Marquardt C., Mai N.: A computational procedure for movement analysis in handwriting. J. Neurosci Methods (1994) 52:39-45

5. Pullmann S.: Spiral Analysis: A New Technique for Measuring Tremor With a Digitizing Tablet, Movement Disorders, vol.13, Suppl.3, (1998), pp85-89

6. Eichhorn T.E., Gasser T., Mai N., Marquardt C., Arnold G., Schwarz J., Oertel W.H. Computational analysis of open loop handwriting movements in Parkinson's disease: a rapid method to detect dopaminergic effects. Mov Disord (1996); 11: 289-297.

7. Ingvarsson P.E., Gordon A.: Coordination of Manipulative Forces in Parkinson's Disease, Exp. Neurology 145, 489-501 (1997)

8. Fellows S., Noth J.: Grip Force Abnormalities in De Novo Parkinson's Disease, Movement Disorders, Vol 19,(5) 560-565 (2003)

9. Fellows S.J., Noth J., Schwarz M. Precision grip and Parkinson's disease. Brain 1998; 121: 1771.1784

10. http://www.bisp-regensburg.de/

11. Steven M.Pincus: Appoximative entropy as a measure of system complexity, Proc.Natl. Acad. Sci. USA Vol. 88, pp. 2297-2301

12. Madalena Costa, Ary L. Goldberger, C.-K. Peng: Multiscale entropy analysis of biological signals, Physical Review E 71, 021906 (2005) 